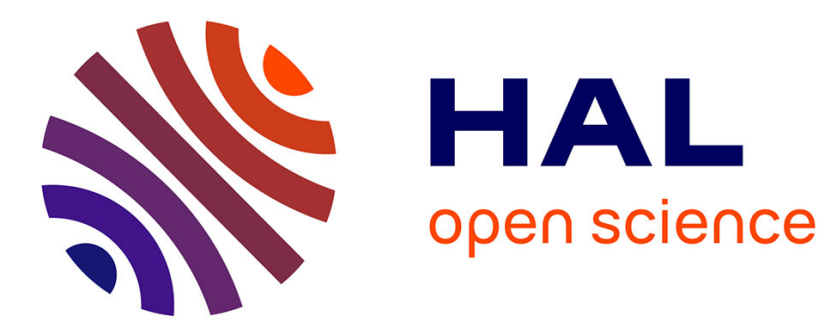

\title{
Usages des modèles spatiaux pour la prospective
}

Thomas Houet

\section{- To cite this version:}

Thomas Houet. Usages des modèles spatiaux pour la prospective. Revue internationale de Géomantique, 2015, 25/1, pp.123-143. hal-01194496

\section{HAL Id: hal-01194496 \\ https://hal-univ-tlse2.archives-ouvertes.fr/hal-01194496}

Submitted on 7 Sep 2015

HAL is a multi-disciplinary open access archive for the deposit and dissemination of scientific research documents, whether they are published or not. The documents may come from teaching and research institutions in France or abroad, or from public or private research centers.
L'archive ouverte pluridisciplinaire HAL, est destinée au dépôt et à la diffusion de documents scientifiques de niveau recherche, publiés ou non, émanant des établissements d'enseignement et de recherche français ou étrangers, des laboratoires publics ou privés. 


\title{
Usages des modèles spatiaux pour la prospective
}

\author{
Houet Thomas
}

GEODE UMR 5602 CNRS, Université Toulouse le Mirail

5 allée Antonio Machado, 31058 Toulouse cedex

thomas.houet@univ-tlse2.fr

\begin{abstract}
RESUME.
Cet article théorique a pour objectif de faire un état de l'art sur l'usage des modèles spatiaux pour la prospective. Dans un premier temps, il présente un bref historique de la convergence implicite entre prospective et géographie. Dans un second temps, il aborde la question du choix du modèle en présentant les critères à prendre en compte. Dans un troisième temps, il présente la validation des modèles comme un moyen d'améliorer la plausibilité des scénarios à travers la combinaison de méthodes d'évaluation. Enfin, si on constate un usage de plus en plus important de modèles spatiaux en prospective, les méthodes évoquées sont loin d'être exhaustives et replacent la géoprospective comme une simple communauté de pratiques et de méthodes ayant un objectif commun: mieux explorer les futurs pour éclairer l'action présente. Au final, il apporte un éclairage sur l'apport des modèles aux démarches prospectives et vise à aider les géographes, les modélisateurs et/ou les prospectivistes à choisir un modèle approprié à leur problématique et à leurs objectifs afin de tirer parti de tous les avantages qu'ils offrent. Il tente également de clarifier certaines confusions sémantiques qui existent autour de l'usage des modèles couplés à des scénarios.
\end{abstract}

ABSTRACT.

This theoretical paper presents a state of the art on the use of spatial models dedicated to prospective studies. The first part summarizes the converging interests of foresight studies and geography. The second part gives helpful insights to answer the question of how choosing an appropriate model for exploring the future of territories/landscapes. The third part is dedicated to models validation tools and methods which combination may be useful to improve the plausibility of scenarios. These methods are not exhaustively described and illustrate that the geoprospective consists in a community of practices and tools with a common objective: better exploring the future to improve current decision making. It aims at highlighting the contribution of models for such purpose and helping geographer, modeler and/or prospectivists to choose a convenient model. It attempts to clarify some misunderstanding and confusions that already exists when combining scenarios and models.

MotS-CLES: Typologie, Scénarios, Simulation dynamique et spatialement explicite, Validation, Plausibilité.

KEYWORDS: Typology, Scenarios, Spatially explicit and dynamic simulation, Validation, Plausibility 


\section{La place des modèles spatiaux dans une démarche prospective}

\subsection{Prospective et géographie, une convergence implicite}

La prospective a connu un réel essor au début des années 1970 grâce au rapport Meadows (Meadows et al., 1972) qui a fait émerger le concept de développement durable. Dans cet ouvrage, un modèle de simulation (World 3) était utilisé pour prendre en compte les interactions entre évolution de la démographie, des ressources et des déchets. La généralisation de ce concept aux projets d'aménagement du territoire a contribué, par incidence, à accroitre l'usage de la prospective dans ce domaine.

Une convergence implicite entre géographie et prospective s'est opérée avec la dimension spatiale comme dénominateur commun de représentation de la complexité. Dès 1971, la géographie s'est intéressée à l'exploration à long terme du futur (Phlipponneau, 1971). Dans le même temps, la prospective territoriale émerge dans le domaine de l'aménagement et de la planification du territoire, portée par la DATAR (Datar, 1971a; 1971b), illustrant cette convergence. La prospective territoriale est définie comme ayant pour objet de définir des faits et des territoires, porteurs d'avenir, dans une perspective opérationnelle programmatique (Delamarre, 2002). La dimension spatiale prend une part importante, sans pour autant être centrale, l'espace étant envisagé plus comme un support pour représenter des phénomènes, notamment socio-économiques, que de façon systémique tenant compte des interactions spatiales et des jeux d'échelles imbriquées (Emsellem et al., 2012). Les travaux de l'Atelier Parisien d'Urbanisme (APUR, 1990) ou du Conseil économique et social de Midi-Pyrénées (CES, 2003) en sont des exemples. Par la suite, les développements de méthodes et d'outils suivant le paradigme de l'analyse spatiale a également contribué à renforcer cette convergence. L'analyse systémique, les méthodes de formalisation de la complexité et de simulation ont ainsi permis de renforcer la place de la dimension spatiale dans le processus prospectif (Voiron, 2012 ; Emsellem et al., 2012).

La géoprospective (Houet, 2006 ; Voiron, 2006) constitue ainsi une communauté de pratiques visant à mettre la dimension spatiale, sous toutes ses acceptions, au cœur de la démarche prospective, depuis la caractérisation des dynamiques spatiales et temporelles du système, l'intégration des interactions multi-échelles jusqu'à la simulation spatialement explicite des changements futurs possibles pour l'évaluation qualitative et quantitative de leurs impacts. Si la prospective repose sur deux piliers fondamentaux d'un point de vue méthodologique, la scénarisation et une approche participative (Houet et Gourmelon, 2014; Etienne, 2012), les modèles géographiques ou spatialement explicites sont ainsi la pierre angulaire de la géoprospective, faisant le lien entre « scénarios » et «participation ».

Parallèlement, les travaux réalisés au sein de la communauté internationale LUCC (Land use and cover changes) ont largement contribué au développement de modèles de simulation spatiale en se focalisant notamment sur les changements d'occupation et d'usages de sols à diverses échelles (Lambin et Geist, 2006). Il ne 
s'agit pas ici de citer l'ensemble des modèles ni-même les diverses typologies de modèles (Coquillard et Hill, 1997 ; Agarwal et al., 2000) existants. Néanmoins, les automates cellulaires, les modèles à base d'agents, intégrés ou non dans des plateformes de modélisation, constituent les méthodes et outils fondamentaux pour intégrer et simuler des dynamiques spatio-temporelles de façon spatialement explicite. Les projets internationaux LAND ${ }^{1}$, ainsi que son prédécesseur $\mathrm{LUCC}^{2}$, ont ainsi permis, riches des travaux de recherches et développements méthodologiques, de faire émerger la science du land change (Turner et al., 2007; Dearing et al., 2010 ; Van der Leeuw et al., 2011) ou encore la théorie du Land System (Kok et al., 2004) où «modèle, scénario et acteur » doivent être appréhendés de concert pour comprendre les changements passés et anticiper ceux à venir. Leur finalité vise toujours l'évaluation quantifiée des changements et de leurs impacts possibles au travers le prisme de la dimension spatiale (surfaces, localisation, etc.) ainsi que l'aide à la décision pour une gestion à moyen ou long terme des ressources (Gourmelon et al., 2008).

Néanmoins, dans la pratique, des confusions sémantiques et/ou des déséquilibres dans le couplage des approches inhérentes à la modélisation, à la construction de scénarios ou encore à la participation, persistent. Ainsi, par exemple, le résultat d'une simulation est parfois décrit comme une simulation prospective, sans pour autant répondre à certains préceptes méthodologiques, tels que ceux relatifs à la méthode des scénarios décrite par Godet (1986). Ceci peut engendrer un effet contre-productif de la prospective, tel qu'un rejet des scénarios produits par les principaux concernés par exemple (Godet and Roubelat, 1996 ; Durance and Godet, 2010). Cet article a pour objectif d'apporter une clarification terminologique, au regard des approches méthodologiques propres à la prospective et la modélisation, en mettant en lumière le rôle respectif des scénarios et des modèles.

\subsection{Scénarios prospectifs et modèles : usages, fonctions et objectifs}

Le couplage entre prospective et modèle renvoie toujours à la question de l'usage du modèle, et de la dépendance de l'un vis-à-vis de l'autre. En d'autres termes, le scénario prospectif alimente-t-il le modèle ou bien est-il le résultat de la simulation?

Du point de vue de la prospective, les modèles peuvent être assimilés à une méthode, parmi d'autres, destinée à explorer le futur. Toutefois, la complexité des interactions à prendre en compte pour faire évoluer le système territorial étudié peut rendre inopérant, inadapté, incomplet, la plupart des modèles. En effet, étant par définition une simplification de la réalité, ils ne permettent pas d'intégrer les particularismes locaux. Seul l'exercice intellectuel permet de prendre en compte l'ensemble des processus et interactions entre des facteurs sociaux, démographiques, politiques, technologiques, économiques, culturels, historiques, géographiques ou

\footnotetext{
${ }^{1}$ LAND project : http://www.globallandproject.org/

${ }^{2}$ http://www.igbp.net/4.1b8ae20512db692f2a680009062.html
} 
encore environnementaux (Huss et Honton, 1983 ; 1987). De plus, de par leur architecture et la formalisation préalable qu'ils requièrent, les modèles peuvent présenter certaines limites pour construire et explorer une plus grande diversité de situations futures contrairement à d'autres méthodes de prospective fondées sur une approche systémique, intellectuelle, logique et qualitative (par opposition aux modèles). Ainsi, il existe une multitude de méthodes mobilisables pour explorer le futur, les scénarios étant l'une des plus répandues. Trois grandes 'écoles' de construction de scénarios, au niveau international, se distinguent (Amer et al., 2013 ; Bradfield et al., 2005) :

- l'école des 'intuitive logics' (Wack, 1985a ; 1985b). Cette école est fondée sur les relations logiques de causalité entre les variables du système et n'utilise pas de modèles ou d'algorithmes mathématiques. Les approches méthodologiques sont nombreuses, la plus populaire et répandue étant celle proposée par le Stanford Research Institute International (Huss, 1988).

- l'école des 'probabilistic modified trends'. Elle se distingue par les méthodes utilisées: les trends impact analysis et le cross impact analysis (Bishop et al. 2007), méthodes probabilistes servant à projeter des tendances à partir des tendances passées, à déterminer les principales relations de causalités entre des variables, et ainsi de modifier certaines extrapolations.

- l'école française - la prospective (Godet, 1986). Cette dernière, considérée comme une méta-méthode, englobe les deux écoles précédentes (Bradfield et al., 2005) en s'appuyant sur un panel d'outils non délimité et une démarche méthodologique constituée d'étapes spécifiques dont le nombre est variable selon les auteurs (Durand, 1972 ; Godet, 1986 ; Schwartz, 1996 ; Schoemaker, 1993 ; 1995 ; Mermet et al., 2003). Toutefois, que les méthodes utilisées soient quantitatives ou qualitatives, les étapes restent globalement identiques dans les trois écoles citées : (1) la construction de la base regroupe l'identification des variables internes et externes au système étudié (les facteurs de changement), la caractérisation de la dynamique passée du système et les enjeux futurs ; (2) la construction des scénarios sous la forme de cheminements (description de la dynamique future du système) et d'images (description du système à diverses dates clefs) reposant sur la définition d'hypothèses (haute ou basse par exemple) pour chacun des facteurs identifiés préalablement. Une troisième étape, l'action, distingue la prospective «à la française » des deux écoles précédentes, qui se limitent à la construction des scénarios. Après évaluation et comparaison des conséquences des scénarios, cette étape doit permettre de définir des préconisations, règles ou encore mesures favorisant la gestion du système par les acteurs.

La déclinaison de la prospective, quel que soit l'école, en géographie visant à intégrer la dimension spatiale tout au long de la démarche prospective (Houet et al., 2008 ; 2010 ; 2011), confère aux modèles de simulation spatiale une place relativement limitée. Néanmoins, ils permettent de prendre en compte des interactions spatiales que la prospective territoriale n'intègre pas. Leur principal usage consiste à retranscrire spatialement les changements futurs d'occupation et 
d'usages des sols du territoire étudié tels que définis par des scénarios prospectifs à des fins d'évaluation. Toutefois, plusieurs facteurs peuvent influer sur le choix du modèle à utiliser : le type de scénarios, la compréhension du modèle et de son fonctionnement, etc. Le modèle peut également avoir plusieurs fonctions (outil de diagnostic, de co-construction, etc.) et contribuer de diverses façons à l'exercice de prospective. Enfin, son choix va également dépendre des objectifs poursuivis par la démarche prospective. Piveteau (1995) distingue trois types d'objectifs : l'apport de la connaissance sur le futur (simuler des évolutions tendancielles, anticiper des ruptures, explorer des conjectures), faciliter la médiation entre connaissance et action (favoriser le processus d'apprentissage de façon organisée, partagé par tous, de façon plus ou moins ludique et évolutif) et aider à l'action (fournir des données quantifiées menant à des décisions transparentes, consensuelles ou négociées, innovantes et audacieuses). Ainsi, les modèles par leur usage, leur explicitation et les simulations vont pouvoir contribuer à répondre aux objectifs de la prospective.

\subsection{La notion de validation en prospective : la place des modèles}

La question de la validation des projections est aussi une question récurrente à laquelle il est difficile de répondre. En effet, comment peut-on valider des scénarios portant sur le futur? En prospective, cette question se décline différemment, l'objectif étant d'explorer une diversité des futurs possibles et non de tenter de prédire quel sera le futur (Godet, 1977). La validation des scénarios produits est indispensable et consiste à évaluer la démarche de construction et l'apport des scénarios in fine. Elle repose sur des critères dont le nombre peut varier selon les auteurs (Wilson, 1998 ; Alcamo et Henrichs, 2009 ; Durance et Godet, 2010) et vise à vérifier que les scénarios soient caractérisés par la vraisemblance, la cohérence, la pertinence, l'utilité et l'originalité. Piveteau (1995) propose également de rajouter les principes de «vérité et rigueur», « démocratie » et «d'aventure ». Dans le cas de l'utilisation d'un modèle spatial en prospective, la démarche de validation du modèle utilisé ne consiste pas à évaluer le pouvoir prédictif du modèle mais bien à démontrer que ce modèle permet de respecter les critères d'évaluation cités cidessus. C'est la question de la confiance que l'acteur (modélisateur, décideur...) peut avoir dans le modèle qui est soulevée.

Au final, cet article a également pour objectif de faire un état de l'art des usages des modèles utilisés en géographie dans une démarche de prospective, en se focalisant plus spécifiquement sur les modèles de simulation spatiale.

\section{Le choix du modèle spatial}

Le choix du modèle est déterminant et doit être réalisé tôt dans la démarche prospective. Il doit être fait selon les objectifs visés, c'est-à-dire l'apport que l'on attend du modèle et son aptitude à simuler dans le temps et dans l'espace les dynamiques considérées. Ce choix peut être d'autant plus difficile que l'on attend du modèle qu'il réponde à plusieurs objectifs. 


\subsection{Les divers objectifs liés à l'usage de modèles en prospective}

D'après la littérature, si l'on considère les modèles tenant compte d'une dimension spatiale dans leur définition la plus large, plusieurs types d'apports peuvent être distingués :

- Traduire sous la forme de rendus spatiaux, les images d'un scénario à différentes dates. A travers la prospective territoriale, c'était l'objectif premier de l'usage de ces modèles. Divers modèles géographiques peuvent être utilisés, illustrés par les exemples suivants: chorèmes, croquis paysagers (Michelin, 2000), modèles de représentation paysagère virtuelle (Gaucherel et al., 2010), cartographie SIG ou issues de modèles de simulation dynamique (Houet et al., 2008).

- Favoriser la participation et l'apprentissage collectif lors de la construction de scénarios. L'usage de toute représentation simplifiée de la réalité pour identifier les dynamiques du système, les enjeux prospectifs, ou encore les jeux d'acteurs et les processus d'usages des sols lors d'une démarche prospective (construction de la base, définition de scénarios, etc.) peut ainsi constituer un objectif lié à l'usage du modèle. Toutes les méthodes de diagnostic territorial peuvent ainsi être considérées (Lardon et al., 2006 ; Lardon et Roche, 2008 ; Batton-Hubert et al., 2008) tels que, par exemple, les chorèmes (Chery et Smektala, 2003; Lardon et Capitaine, 2008), les croquis paysagers et autre représentation virtuelle des paysages (Michelin, 2000 ; Joliveau et Michelin, 2001 ; Joliveau et Dupuis, 2006) ont ainsi déjà été utilisés à cette fin. La modélisation d'accompagnement peut également offrir des principes méthodologiques particulièrement bénéfiques (Etienne, 2010 ; 2012) afin de bien prendre en compte tous les acteurs, leurs stratégies d'usages ou encore favoriser leur implication dans le processus prospectif, à l'aide de méthodes diverses (méthode ARDI, jeux...) favorisant la coconstruction. L'explication du fonctionnement du modèle de simulation spatiale peut également favoriser la définition collective des processus et zones présentant des enjeux de préservation à l'avenir (Dodane et al., 2011).

- Simuler de façon réaliste les processus spatio-temporels contribuant à l'évolution des territoires et leurs interactions. L'objectif des modèles concernés, principalement dynamiques et spatialement explicites, est de reproduire le plus fidèlement possible les processus d'occupation et d'usage des sols en tenant compte ou non des interactions entre l'agent et son environnement (le territoire), voire les interactions entre agents. A titre d'exemples, cela va de la simulation de la diffusion des innovations technologiques (Daudé, 2004), de l'étalement et de la morphologie urbaine (Dubos-Paillard et al., 2003) voire de réseaux de villes (Perret et al., 2010) ou encore des successions culturales en agronomie des territoires (Houet et Gaucherel, 2007). Les automates cellulaires, les modèles à base d'agents ou encore les plateformes de modélisation constituent les types de modèles majoritairement utilisés. Un fort réalisme renforcera la plausibilité des scénarios prospectifs, en terme de rendu et d'évaluation. 
- Contribuer à l'évaluation des scénarios. La simulation spatiale de changements futurs a pour principal intérêt d'identifier les espaces où ils pourraient se produire à l'avenir. Les cartes ainsi produites peuvent permettre d'identifier des espaces porteurs d'enjeux en matière de gestion / préservation. Les simulations peuvent également permettre d'évaluer une incertitude quant à la probabilité que le changement intervienne à l'avenir. Dans le cas d'un scénario couplé à un modèle présentant des règles aléatoires, des simulations monte carlo rendent possible l'estimation de l'occurrence du changement. Dans le cas de plusieurs scénarios couplés, le croisement du résultat issu de chacun d'eux peut permettre de déterminer les zones présentant une forte probabilité qu'un changement se produise (ex : abandon de terres agricoles) dès qu'il intervient dans plusieurs scénarios (Verburg et al., 2010). Enfin, ces cartes constituent les données d'entrée indispensables à certains modèles ayant pour objectif d'évaluer l'impact de la composition et de la configuration du paysage et de ces changements futurs sur l'environnement (climat, qualité de l'eau, biodiversité, etc.).

- Contribuer à la gestion optimisée de ressources. Dans ce cadre, les modèles utilisés ont pour objectif de trouver la composition ou la configuration paysagère (au sens de l'écologie du paysage) optimale sous contrainte d'une ressource existante (eau, biodiversité, etc.). Ainsi, ils permettent d'évaluer l'influence des mesures d'incitation ou de gestion sur les modes d'occupation et d'usages des sols, et par corollaire, leur faisabilité (Leenhardt et al., 2004 ; Bamière et al., 2011).

Au final, le choix du modèle doit s'effectuer à la lumière des objectifs visés qui peuvent se distinguer à partir des questions suivantes. Le modèle a-t-il pour objectif :

- de représenter le territoire et son évolution de façon simplifiée ou réaliste en termes de résolution, de complexité, de localisation?

- de favoriser la participation ou d'affiner l'évaluation?

- d'évaluer l'influence de l'interaction de multiples processus sur le devenir du territoire ou bien d'évaluer les processus à mettre en ouvre pour atteindre une situation donnée?

\subsection{L'aptitude du modèle à simuler les dynamiques spatiales désirées / définies}

Dès lors que l'on s'intéresse plus spécifiquement aux modèles dynamiques et spatialement explicites, le choix du modèle doit également être effectué d'après son aptitude à simuler des dynamiques spatiales et temporelles spécifiques ou encore à intégrer des interactions entre ressources et acteurs. La détermination de la 'base' doit permettre d'identifier précisément l'ensemble des règles à modéliser et qui auront une influence sur l'évolution du territoire. La représentation cartographique, en mode raster ou vectoriel, se traduit par la simulation de changements de l'état (attribut) et/ou de la forme (géométrie) des unités spatiales élémentaires. La majeure partie des modèles utilisent une représentation raster du territoire, les changements 
de formes étant induits par la juxtaposition dans l'espace de changements attributaires.

Une approche comparative est indispensable pour évaluer l'aptitude d'un modèle de simulation spatiale à simuler les dynamiques spatiales et temporelles qui vont affecter l'évolution d'un territoire (Houet et Gaucherel, 2007). Il faudra ainsi s'assurer que le modèle est adapté à représenter la complexité du système étudié dans ses dimensions spatiale (unité spatiale élémentaire, étendue, interactions spatiales), temporelle (pas de temps, durée, boucles de rétroactions), et humaine (agent, groupes sociaux, relations hiérarchiques) (Agarwal et al., 2000). En effet, des dynamiques et interactions spatiales différentes peuvent être observées suivant les échelles de temps et d'espace considérées, rendant inadaptés certains modèles suivant les thématiques / territoires étudiés (Houet et Hubert-Moy, 2006). Certains peuvent être adaptés à simuler divers processus spatiaux (diffusion, relations multiéchelles, etc.) mais présenter des limites à simuler et/ou combiner des processus présentant une dynamique temporelle continue, discrète et/ou cyclique.

L'évaluation de l'aptitude du modèle peut ainsi conduire à confirmer le choix d'un modèle ou, dans le cas contraire, à développer un modèle spécifique à l'aide de plateformes de modélisation par exemple. D’une façon générale, deux types de modèles de simulation se distinguent, et dont la dichotomie peut aider à l'analyse et au choix du modèle : process-based versus pattern-based modèles (Fortin et al., 2003 ; Houet et al., 2014). Les premiers ont pour objectif de simuler les processus influençant l'évolution d'un paysage (modèles de croissance, de diffusion d'espèces, etc.). Les seconds cherchent à simuler un patron paysager (habitat dispersé, contigu, etc.) qui imite les dynamiques spatiales observées.

\section{La question de la validation du modèle}

Le principe fondamental de la prospective consiste à explorer une multitude de futurs possibles pour éclairer l'action présente (Hatem, 1993). En aucun cas, il s'agit de prédire le futur. Dès lors, la calibration du modèle à partir d'une période passée peut ne pas constituer un réel enjeu en modélisation prospective.

La validation du modèle a pour objectif de renforcer la plausibilité des scénarios. C'est-à-dire démontrer que les dynamiques spatiales sont crédibles et vraisemblables. Cela consiste à s'assurer que la représentation spatiale et temporelle des processus simulés respecte les tendances, les processus de changements voire les patrons paysagers tels qu'ils sont préalablement définis dans les scénarios prospectifs.

\subsection{La place de la calibration des modèles}

La plupart des modèles LUCC probabilistes existant et facilement utilisables s'appuient sur l'utilisation de données (cartes, statistiques...) traduisant une évolution récente, observée. Leur fonctionnement «type » se résume en trois étapes (Mas et al., 2011; 2014) : (1) à partir de ces données, ils estiment les 
tendances de changements futurs en terme de quantité de changements ainsi que les probabilités de transition d'une classe d'occupation du sol vers une autre ; (2) à l'aide de données géographiques traduisant les facteurs explicatifs de la localisation des changements, ils produisent, pour chaque transition et à l'aide de méthodes statistiques plus ou moins complexes (régression logistique, réseau neuronal, etc.), une carte des probabilités de localisation des changements ; (3) ils utilisent des méthodes dynamiques et spatialement explicites (automate cellulaire, système multiagents, etc.) pour simuler les évolutions estimées et les allouer dans l'espace. La calibration d'un modèle à partir de données passées constitue un faux enjeu pour la modélisation prospective, sauf éventuellement dans le cas de scénarios tendanciels, et ce pour plusieurs raisons :

- les dates des données d'entrée ont une influence sur l'estimation des quantités de changements futurs (Houet et Hubert-Moy, 2006). L'estimation des changements futurs (quantité) ne doit pas dépendre du modèle (et par corollaire des données d'entrée disponibles) mais être définie par le scénario prospectif (Fig. 1a);

- le mode probabiliste d'estimation des quantités de changements futurs implique une extrapolation des tendances de façon linéaire dans le temps. En d'autres termes, la quantité totale de changement estimée est attribuée selon des quantités annuelles similaires. Ce type de modèle est donc inadapté dès lors qu'un scénario prospectif prévoit des dynamiques de changements variables au cours du temps. Ainsi, la simulation engendrera une évolution linéaire qui ne sera pas concordante avec le scénario: si l'image finale peut atteindre la situation définie par le scénario, le cheminement pourra être discordant (Fig. 1b) ;

- les probabilités de transition d'une classe d'occupation du sol à une autre sont fixes dans le temps pour ce type de modèle, sauf dans le cas où un scénario prospectif prévoit une modification de ces probabilités. Ainsi, aucune nouvelle transition ne peut être simulée. De plus, le poids des facteurs de changements est supposé constant sur la période de calibration et jusqu'à l'horizon temporel visé. Kolb et al. (2013) ont mis en évidence que ceci n'était jamais démontré même dans le cas où aucun facteur de changement supplémentaire soit intervenu. De plus, les méthodes utilisées peuvent modifier le poids relatif de chacun d'eux dès lors qu'un nouveau facteur est intégré ;

- à partir d'un même jeu de données et de règles, les modèles peuvent également produire des simulations spatio-temporelles différentes en raison des méthodes mobilisées (Mas et al., 2011) et donc engendrer une incertitude propre (Fig. 1c). 

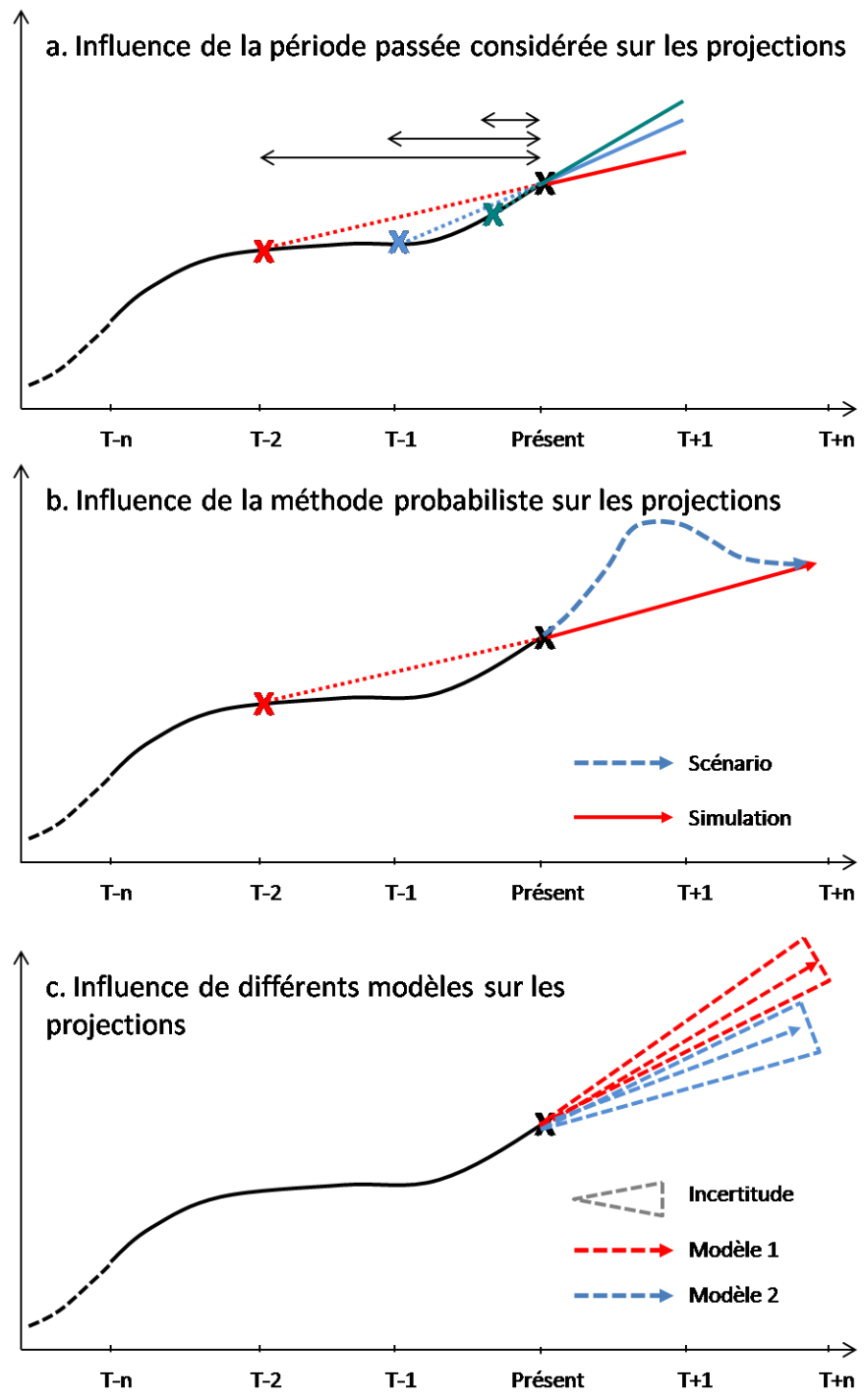

Figure 1. Influence de la calibration et des modèles sur les projections

$\mathrm{Au}$ final, certains modèles de simulation spatiale, tels que les modèles probabilistes appliqués ou non aux changements d'occupation et d'usages des sols, présentent des limites pour spatialiser des scénarios prospectifs présentant des évolutions futures contrastées, ou en rupture par rapport à la période précédente. Dès lors qu'il s'agira de simuler des scénarios tendanciels, leur utilisation devra être faite avec rigueur puisque le résultat des simulations explorant le futur ne pourra pas être vérifié. 


\subsection{La validation pour accrôttre la plausibilité des scénarios prospectifs}

Afin d'accroître la plausibilité des scénarios, la validation consiste à démontrer l'aptitude du modèle à simuler des changements, en termes de dynamiques et de processus, définis par des scénarios. L'approche proposée repose sur la combinaison de plusieurs démarches de validation (Houet et al., 2014) :

- la réalisation de tests de sensibilité et d'intégration. Les premiers permettent de s'assurer que l'ensemble des processus sont convenablement simulés par le modèle. Les seconds s'assurent que l'interaction de ces processus n'entrave pas leur fonctionnement respectif (Houet et Gaucherel, 2007).

- la simulation sur une période passée. Si la calibration sur une période passée peut apparaître comme un «faux enjeu », la démarche de simuler des changements observés durant une période passée (entre $t_{-2}$ et $t_{-1}$ ), et de comparer le(s) résultat(s) de la simulation avec une situation observée (à $t$ ), reste particulièrement intéressante (Houet et Gaucherel, 2007). Dans le cas où le modèle est en mesure de reproduire, sur le plan de la composition et de la configuration du paysage, une évolution semblable à celle qui s'est réellement produite, alors la confiance que l'on peut avoir dans le modèle à produire des situations (par exemple, des paysages) vraisemblables est renforcée.

- la simulation de dynamiques contrastées (en termes de localisation, de quantité et de processus). Si cette démarche est assimilable à des tests d'intégration, elle s'en différencie quelque peu à travers la simulation de scénarios fictifs. L'objectif consiste donc à simuler des ruptures dans les dynamiques de changements (augmentation puis baisse d'une occupation du sol ; modes d'allocation spatiale différents au cours du temps tels que regroupés ou dispersés pour des pattern-based modèles; introduction de nouveaux processus ou arrêt de processus existants pour des process-based modèles...). Si le modèle est en mesure de gérer des dynamiques contrastées, avec un séquençage contrôlé, tout en s'assurant que leurs interactions n'altèrent pas la production de paysages fidèles aux patrons paysagers et/ou aux processus d'usages des sols tels que définis par les scénarios, alors cela renforce la confiance que l'on peut avoir dans le modèle à simuler de façon plausible des scénarios prospectifs.

Il n'existe pas d'indicateur synthétique sous forme statistique ou cartographique qui permette d'évaluer la vraisemblance d'une simulation. De plus, les simulations produites reposent parfois sur des règles aléatoires (choix aléatoire de localisation par exemple), engendrant potentiellement diverses simulations possibles pour un même scénario. Les indicateurs reposant sur la comparaison d'une simulation avec une situation observée tels que le Kappa (Pontius, 2002), la courbe ROC (Pontius and Schneider, 2001) ou encore les indicateurs dérivés des LUCC budgets (Pontius et al., 2004a ; 2004b ; 2008) apparaissent inadaptés dès lors qu'ils sont utilisés individuellement. En effet, ils évaluent le pouvoir prédictif d'un modèle et non son aptitude à correctement simuler les processus d'usages des sols ou les patrons paysagers désirés (Brown et al., 2005) (Tableau 1). Par contre, dans la mesure où chacun de ces indicateurs peut contribuer à évaluer l'aptitude du modèle à simuler soit le motif paysager désiré, soit des dynamiques spatiales ou temporelles 
spécifiques, leur usage combiné permet alors d'accroître la confiance que l'on peut avoir dans le modèle à simuler des scénarios prospectifs. Le cas échéant, c'est-à-dire si ces tests ne sont pas réalisés, il est possible que le résultat de la simulation présente des dynamiques spatiales cohérentes (à travers un motif paysager vraisemblable) et des dynamiques temporelles peu vraisemblables (par exemple, transitions d'occupation des sols incohérentes). Dans ce cas de figure, l'acceptation des sorties des modèles de simulation spatiale peuvent être remis en cause, et par corolaire, les scénarios produits.

Tableau 1. Exemples d'indicateurs utilisables pour valider l'aptitude d'un modèle à simuler de façon vraisemblable des changements d'occupation des sols sur le plan des quantités, des processus ou de la localisation. Les indicateurs utilisés pour les démarches (1) et (2a) sont comparés soit aux paramètres d'entrée du modèle (valeurs attendues après simulation), soit à une situation observée (à la date t). Ceux utilisés pour la démarche (2b) doivent témoigner de valeurs évoluant de façon contrastée (valeurs témoignant de ruptures au cours de l'évolution).

\begin{tabular}{|l|l|l|l|}
\hline \multirow{2}{*}{$\begin{array}{l}\text { Type } \\
\text { de } \\
\text { changement }\end{array}$} & \multicolumn{1}{c|}{$\begin{array}{c}\text { (1) Tests de } \\
\text { sensibilité }\end{array}$} & $\begin{array}{c}\text { (2) Tests d'intégration } \\
\text { (a) Simulation sur une } \\
\text { période passée }\end{array}$ & $\begin{array}{c}\text { (b) Simulation de } \\
\text { tendances contrastées }\end{array}$ \\
\cline { 3 - 4 } $\begin{array}{l}\text { Quantité de } \\
\text { changement }\end{array}$ & $\begin{array}{l}\text { - \% d'occupation } \\
\text { des sols (OS) }\end{array}$ & $\begin{array}{l}\text { - Etat final des \% } \\
\text { d'occupation des sols }\end{array}$ & $\begin{array}{l}\text { - Evolution des \% } \\
\text { d'occupation des sols }\end{array}$ \\
\hline $\begin{array}{l}\text { Processus de } \\
\text { changement }\end{array}$ & $\begin{array}{l}\text { - Cartes } \\
\text { d'occurrences d'une } \\
\text { OS Carte de } \\
\text { localisation }\end{array}$ & $\begin{array}{l}\text { - Evolution des \% } \\
\text { d'occupation des sols } \\
\text { - Cartes d'occurrences } \\
\text { d'une OS } \\
\text { - Indice Kappa } \\
\text { - Comparison Map and } \\
\text { Profile (CMP) }\end{array}$ & $\begin{array}{l}\text { - Evolution des } \% \\
\text { d'occupation des sols } \\
\text { - Cartes d'occurrences } \\
\text { d'une OS } \\
\text { - Kappa, CMP, etc. }\end{array}$ \\
\hline $\begin{array}{l}\text { Localisation des } \\
\text { changements }\end{array}$ & $\begin{array}{l}\text { - Métrique } \\
\text { - Distribution par } \\
\text { rapport à des } \\
\text { éléments pérennes } \\
\text { du paysage }\end{array}$ & $\begin{array}{l}\text { - Cartes d'occurrences } \\
\text { d'une OS } \\
\text { - Kappa, CMP, etc. } \\
\text { - Métrique paysagère } \\
\text { - Distribution par rapport } \\
\text { à des éléments pérennes } \\
\text { du paysage }\end{array}$ & $\begin{array}{l}\text { - Cartes d'occurrences } \\
\text { d'une OS } \\
\text { - Kappa, CMP, etc. } \\
\text { - Métrique paysagère } \\
\text { - Distribution par rapport } \\
\text { à des éléments pérennes } \\
\text { du paysage }\end{array}$ \\
\hline
\end{tabular}

Par exemple, ces techniques ne permettent pas de démontrer que les processus ou encore les dynamiques sont bien respectés. Autre exemple, l'indice d'erreur due à la quantité (Pontius, 2002) peut ne pas être pertinent pour des scénarios prospectifs normatifs, car le modèle est censé simuler une quantité de changement définie. Si une erreur due à la quantité subsiste, cela signifie que le modèle n'est pas apte à correctement simuler ce type de scénario. Pour chacune des démarches mentionnées, 
un ou plusieurs indicateurs peuvent être utilisés: indicateur statistique simple (proportions d'occupations du sol), ROC, Kappa et autre méthodes de validation par comparaison (Pontius, 2002; Pontius et al., 2008) ainsi que les métriques paysagères, telles que des indices d'hétérogénéité ou de connectivité du paysage (Haines-Young et Chopping, 1996 ; Uuermaa et al., 2009), etc. Par exemple, les indices issus du CMP (Comparison Map and Profile - Gaucherel et al., 2008) peuvent permettre de mettre en évidence la similitude d'un motif paysager simulé avec un paysage de référence (Houet et al., 2014). C'est la combinaison de ces indicateurs, à travers ces trois démarches de validation, qui est importante et qui permet de valider l'aptitude du modèle à simuler des dynamiques spatio-temporelles vraisemblables, définies par un ou plusieurs scénarios (Tableau 1). Néanmoins, rappelons que la plausibilité des simulations dépend de la plausibilité des scénarios qui doit être vérifiée préalablement comme cela a été évoqué dans le paragraphe 1.2. Un modèle, une fois validé, peut en tout état de cause simuler de façon vraisemblable un scénario totalement incohérent. Au final, le protocole de validation proposé est (1) spécifiquement adapté aux modèles utilisés dans une démarche prospective et (2) permet d'accroître la plausibilité des scénarios au travers des simulations spatiales.

\section{Influence du type de scénario dans l'usage de modèle}

De nombreuses typologies de scénarios existent dans la littérature (Greeuw et al., 2000 ; Hatem, 1993 ; Poux, 2003). Force est de constater qu'une première typologie distingue les scénarios suivant l'usage de modèles: les scénarios qualitatifs (narratifs) versus quantitatifs (issus de modèles). Dans le contexte d'une prospective urbaine, Marchadier et al. (2012) distinguent ces deux approches en illustrant leurs sorties respectives sous la forme de 'imagined city' vs. 'simulated city'. La méthode de construction des scénarios, distinguant les scénarios exploratoires et normatifs, peut également influer sur le type de modèle spatial utilisé (Houet et al., 2008). Si pour les premiers, l'usage de modèles de simulation spatiale apparait implicite, cela reste moins évident pour les seconds.

Les scénarios exploratoires parcourent le futur au fur et à mesure, sans idées préconçues. Les process-based modèles sont particulièrement adaptés : la somme des règles et des processus, produit des images du futur à un pas de temps défini par le modélisateur, dont la succession dans le temps constitue l'évolution (cheminement). La somme des processus et de leurs interactions spatiales et temporelles peut conduire à des évolutions non linéaires et dont la situation finale constitue l'image du futur. Que les hypothèses retenues soient contrastées ou non (par exemple, évolution démographique, etc.), les scénarios exploratoires permettent d'évaluer les impacts potentiels de facteurs de changements (démographie, changement climatique, la proximité à ...) et de leurs interactions.

Les scénarios normatifs partent d'une image du futur, désirée ou non, et reconstruisent le cheminement de façon rétrospective. L'objectif étant d'identifier les leviers d'actions à mettre en œuvre (ex : règles d'usages des sols) pour arriver à cette situation. Ce type de scénario est particulièrement adapté dans le cas de la 
gestion spatiale d'une ressource, où l'organisation spatiale des types d'occupation des sols aura une forte influence sur la ressource. Par exemple, atteindre une mosaïque paysagère présentant tel niveau de fragmentation, d'hétérogénéité ou de connectivité peut être essentiel pour le maintien d'une biodiversité faunistique. Les modèles spatiaux visant à produire les images du futur sont nombreux (SIG, chorèmes, etc.). Les modèles de simulation spatiale permettant de spatialiser des scénarios normatifs peuvent être distingués en deux catégories selon les objectifs de rendus paysagers poursuivis (Houet et al., 2014) : (1) lorsque l'objectif porte seulement sur la composition paysagère (quantité de changement à atteindre), les process-based et pattern-based modèles sont adaptés. Ils allouent spatialement les changements jusqu'à atteindre les proportions d'occupation et d'usages des sols attendus (Engelen et al., 2007 ; Verburg et al., 2008) ; (2) lorsque l'objectif repose sur la configuration spatiale (une organisation spatiale à atteindre), les pattern-based modèles sont adaptés dès lors qu'ils reposent sur des méthodes d'optimisation (Bamière et al., 2011; Gusdorf et Hallegatte, 2007; Gusdorf et al., 2008). La difficulté est réelle dès lors que l'objectif consiste à reproduire une somme de processus cohérents - un cheminement - et une configuration spatiale désirée - une image du futur. Pour ce faire, il s'agit de coupler des méthodes d'optimisation avec un process-based modèle : les méthodes d'optimisation permettent d'atteindre une situation prédéfinie (à l'équilibre, désirée ou non) à partir d'une somme de processus spatio-temporels (par exemple, les changements d'occupation des sols tels que des rotations culturales en milieu agricole). Ce type de modèle est beaucoup plus rare (Benke et al., 2011 ; Houet et al., 2014).

Dans tous les cas, si la localisation des changements futurs reste incertaine, elle doit être vraisemblable en termes de dynamique(s), de composition(s) et de structure(s) paysagères. Ainsi, dès qu'une étude prospective visant à spatialiser l'évolution des territoires est envisagée, il est nécessaire de savoir quelle est la place du modèle dans le processus prospectif : les simulations issues du modèle utilisé constituent-elles les scénarios prospectifs ou à l'inverse, le scénario prospectif défini préalablement fournit-il aux modèles tous les paramètres d'entrée du modèle ? Dans la pratique, le choix in fine du modèle est le fruit d'un processus itératif entre scénario et modèle durant la démarche prospective, qui découle d'un compromis entre la gamme d'incertitude que l'on souhaite explorer dans le futur, c'est-à-dire le type de scénario choisi, et les aptitudes du modèle à simuler les processus en jeu. Au final, on peut distinguer des démarches de prospective qui reposent sur des approches «modèle-dépendantes » ou « scénario-dépendantes ». Pour les premières, des hypothèses générales sont arrêtées, alimentant un modèle choisi de façon rigoureuse, et dont les simulations constituent le scénario prospectif. Les secondes prévoient la définition de scénarios desquels devront être déterminés les paramètres d'entrée du modèle. Les simulations ainsi produites illustreront le scénario à l'aide de variables quantitatives. Cette dernière approche permet d'explorer une plus grande diversité de futurs. 


\section{La géoprospective : une communauté de pratiques et de méthodes}

La convergence entre prospective et géographie est évidente et implicite depuis quelques décennies. La modélisation prospective a été identifiée comme un enjeu scientifique et méthodologique fort par le CNRS dans le plan stratégique 2003-07 du de la section 39 (Espaces et Territoires), dans le projet de l'InSHS (2009, p.4) ainsi que lors de la «Prospective de l'Institut Ecologie et Environnement» de 2009 (InEE, 2009). Fondamentalement pluridisciplinaire, elle a bénéficié des avancées et des méthodes réalisées dans le domaine de la prospective, de la modélisation et de la géographie. La géoprospective est née de cette effervescence et constitue une communauté de pratiques et de méthodes visant à l'amélioration de l'exploration du futur (Houet et Gourmelon, 2014). Si le terme même de géoprospective fait débat (Ensellem et al., 2012) et que sa définition reste floue car il ne s'agit pas d'une science mais plutôt d'un concept proposé par des géographes, elle propose des méthodes et approches, où l'analyse et la modélisation spatiale jouent un rôle central, permettant de mieux prendre en compte la dimension spatiale tout au long $\mathrm{du}$ processus prospectif. Celles évoquées sont loin d'être représentatives d'un recensement exhaustif des méthodes mobilisables. D'autres, venant par exemple d'autres champs disciplinaires, vont probablement émerger dans les années à venir et pourront être mobilisables. L'objectif réside in fine dans une meilleure prise en compte de la dimension spatiale dans les études prospectives.

De nombreuses confusions existent, tant les termes de scénarios et de modèles sont polysémiques. Les typologies de modèles et de scénarios sont également nombreuses et ne permettent pas toujours d'évaluer l'aptitude d'un modèle à spatialiser tel ou tel scénario. Ils pâtissent également d'un effet de mode : l'usage de ces termes n'est pas réalisé de façon rigoureuse par rapport au cadre méthodologique auxquels ils se réfèrent. A travers cet article, l'objectif aura été de clarifier certains aspects sémantiques et méthodologiques, et visant à éclairer, dans leur choix et selon la place qui leur est réservée, les futurs utilisateurs de modèles à des fins prospectives.

\section{Remerciements}

Cet article a bénéficié du soutien du projet ANR 2010 JCJC 180401 «ODE RESPYR» (http://w3.mode-respyr.univ-tlse2.fr/index.php).

\section{Bibliographie}

Agarwal C., Green G.L., Grove M., Evans T., Schweik C., (2000). A review and assessment of land-use change models : Dynamics of space, time and human choice, General Technical Report NE-297, U.S. Department of Agriculture, Forest Service.

Amer M., Daim T.U., Jetter A., (2013). A review of scenario planning. Futures, vol. 46, p. 23-40.

Alcamo T., Henrichs T., (2009). Towards guidelines for environmental scenario analysis, Environmental Futures: The Practice of Environmental Scenario Analysis, first ed., Elsevier, Amsterdam, The Netherlands, p. 13-35. 
APUR, Direction Régionale de l'équipement d'Ile-de-France, Institut d'aménagement et d'urbanisme de la Région Ile-de-France (1990). Livre blanc de l'Ile de-France. Paris : APUR, DREIF, IAURIF.

Bamière L., Havlík P., Jacquet F., Lherm M., Millet G., Bretagnolle V., (2011). Farming system modelling for agri-environmental policy design: the case of spatially nonaggregated allocation of conservation measures. Ecological Economics, vol. 70, iss. 5, p. 891-899.

Batton-Hubert M., Joliveau T., Lardon S., (2008). Modélisation spatiale et décision territoriale participative. Conception et mise en œuvre dans des ateliers chercheursacteurs. Revue Internationale de Géomatique, vol. 18, n 4, p. 549-569.

Benke K.K., Wyatt R.G., Sposito V.A., (2011). A discrete simulation approach to spatial allocation of commodity production for revenue optimisation over a local region. Journal of Spatial Science, vol. 56, iss. 1, p. 89-101.

Bishop P., Hines A., Collins T., (2007). The current state of scenario development: an overview of techniques, Foresight, vol. 9, p. 5-25.

Bradfield R., Wright G., Burt G., Cairns G., Van Der Heijden K., (2005). The origins and evolution of scenario techniques in long range business planning, Futures, vol. 37, p. 795-812.

Brown, D.G., Page, S., Riolo, R., Zellner, M., Rand, W., (2005). Path dependence and the validation of agent-based models of land use. International Journal of Geographical Information Science, vol. 19, iss. 2, p. 153-174.

Chéry J.P., Smektala G., (2003). Construire un modèle graphique spatial pour développer un modèle de simulation : quel apport pour la gestion des ressources en ligneux ? Actes du colloque international "Organisation spatiale et gestion des ressources et des territoires ruraux », 25-27 février 2003, Montpellier, France.

CES de la Région Midi-Pyrénées (2000). Les desseins de 2030. Une prospective pour les habitants de Midi-Pyrénées. Paris : Futuribles International, INRA, Coll. «travaux et recherches prospectives $»$.

Coquillard P., Hill D.R.C., (1997). Modélisation et simulation d'écosystèmes. Des modèles déterministes aux simulations à évènements discrets, Masson, Paris.

DATAR (Délégation à l'Aménagement du Territoire et à l'Action Régionale). (1971a). Scénarios d'aménagement du territoire. Essais méthodologiques. Paris : La documentation française, collection Travaux et Recherches de Prospective.

DATAR (Délégation à l'Aménagement du Territoire et à l'Action Régionale). (1971b). Une image de la France en l'an 2000. Scénario de l'inacceptable. Paris : La documentation française, collection Travaux et Recherches de Prospective.

Daudé E., (2004). Apports de la simulation multi-agents à l'étude des processus de diffusion », Cybergéo, n²55, http://cybergeo.revues.org/3835

Dearing J.A., Braimoh A.K., Reenberg A., Turner B.L., Van der Leeuw S., (2010). Complex land systems: the need for long time perspectives to assess their future. Ecology and Society, vol. 15, n 4 , p. 21.

Delamarre A. (2002). La prospective territoriale. Paris : La documentation française. Collection Territoire en mouvement. 
Dodane C., Joliveau T., Honneger A., (2011). Un atelier de géoprospective sur l'évolution de l'occupation du sol dans le bassin versant de l'Yzeron à horizon 2030, Acte du séminaire Géoprospective : Apports de la dimension spatiale aux recherches prospective, 4-5 avril 2011, Nice.

Dubos-Paillard E., Guermond Y., Langlois P., (2003). Analyse de l'évolution urbaine par automate cellulaire : le modèle SpaCelle, L'espace géographique, vol. 4, p. 357-378

Durand J. (1972). A new method for constructing scenarios, Futures, vol. 4, p. 325-330.

Durance P., Godet M., (2010). Scenario building: uses and abuses. Technological forecasting and social change, vol. 77 , p. 1488-1492.

Emsellem K., Liziard S., Scarella F., (2012). La géoprospective: l'émergence d'un nouveau champ de recherche? L'espace Géographique, vol. 12, p. 154-168.

Engelen, G., Lavalle, C., Barredo, J.I., Meulen, M. and White, R. (2007). The MOLAND modelling framework for urban and regional land-use dynamics. Chapter 17. Modelling land-use change; progress and applications. Springer, Dordrecht, p. 297-319.

Etienne M. (2010). La modélisation d'accompagnement: une démarche en appui au développement durable. Quae editions, Paris.

Etienne M. (2012). La modélisation d'accompagnement: une forme particulière de géoprospective, L'espace Géographique, vol. 12, p. 128-137.

Fortin M.-J., Boots B., Csillag F., Remmel T.K., (2003). On the role of spatial stochastic models in understanding landscape indices in ecology, Okois, vol. 102, n 1, p. 203-212.

Gaucherel C., Griffon S., Misson L., Houet T., (2010). Combining process-based models for future biomass assessment at landscape scale, Landscape Ecology, vol. 25, iss. 2, p. 201215.

Gaucherel, C., Alleaume, S., Hély, C., (2008). The Comparison Map Profile method: a strategy for multiscale comparison of quantitative and qualitative images. IEEE Transactions on Geoscience and Remote Sensing, vol. 46, iss. 9, p. 2708-2719.

Godet M. (1977). Crise de la prévision, essor de la prospective, Presses Universitaires de France.

Godet M. (1986). Introduction to la prospective: seven key ideas and one scenario method. Futures, vol. 18, p. 134-157.

Godet M., Roubelat F., (1996). Creating the future: the use and misuse of scenarios. Long Range Planning, vol. 29, iss. 2, p. 164-171.

Gourmelon F., Etienne M., Rouan M., Kerbiriou C., Charles M., Bioret F., Chlous-Ducharme F., Guermeur Y., Levrel H., (2008). Éléments de prospective environnementale dans une réserve de biosphère, Cybergeo, $\mathrm{n}^{\circ}$ 429, http://cybergeo.revues.org/20343

Greeuw S.C.H., van Asselt M.B.A., Grosskurth J., Storms C.A.M.H., Rijkens-Klomp N., Rothman D.S., Rotmans J., (2000). Cloudy crystal balls: an assessment of recent European and global scenario studies and models, European Environment Agency, Copenhagen, Denmark.

Gusdorf F., Hallegatte S., (2007). Compact or spread-out cities: Urban planning, taxation, and the vulnerability to transportation shocks. Energy Policy, vol. 35, iss. 10, p. 4826-4838. 
Gusdorf F., Hallegatte S., Lahellec A., (2008). Time and space matter: How urban transitions create inequality. Global Environmental Change, vol. 18, iss. 4, p. 708-719.

Haines-Young R., Chopping M., (1996). Quantifying landscape structure: a review of landscape indices and their application to forested landscapes, Progress in Physical Geography, vol. 20, iss. 4, p. 418-445.

Hatem F. (1993). La prospective : Pratiques et méthodes, Economica, série "Gestion", Paris.

Houet T. (2006). Modélisation prospective de l'occupation du sol en zone agricole intensive : Evaluation par simulations dynamiques de l'impact de l'évolution des exploitations agricoles dans la France de l'Ouest, Norois, n¹98, 2006/1, p. 35-47

Houet T., Hubert-Moy L. (2006). Modelling and projecting land-use and land-cover changes with a cellular automaton considering landscape trajectories: an improvement for simulation of plausible future states, EARSeL eProceedings, n ${ }^{\circ} 5$, p. 63-76

Houet T., Gaucherel C., (2007). Simulation dynamique et spatialement explicite d'un paysage agricole bocager : Validation sur un petit bassin versant breton sur la période 1981-1998, Revue Internationale de Géomatique, vol. 17, n³-4, p. 491-516

Houet T., Hubert-Moy L., Tyssot C., (2008). Modélisation prospective spatialisée à l'échelle locale : approche méthodologique, Revue Internationale de Géomatique, vol. 18, n³, p. 345-373.

Houet T., Loveland T. R., Hubert-Moy L., Napton D., Gaucherel C., Barnes C., (2010). Exploring subtle land use and land cover changes: a framework based on future landscape studies, Landscape Ecology, vol 25, iss. 2, p.249-266.

Houet T., Hubert-Moy L., Tyssot C., (2011). Fine scale spatialised prospective modelling - a methodological approach. Application to water management in Brittany, Revue Internationale de Géomatique, vol. 21, p. 67-93.

Houet T., Gourmelon F., (2014) La géoprospective - Apport de la dimension spatiale aux approches prospectives, Cybergéo, n 667 , http://cybergeo.revues.org/26194

Houet T., Schaller N., Castets M., Gaucherel C., (2014). Improving the simulation of fine scale landscape changes: A combined process-based / optimization approach. International Journal of Geographical Information Science, In press.

Huss W.R. (1988). A move toward scenario analysis, International Journal of Forecasting, vol. 4, p. 377-388.

Huss W.R., Honton E.J., (1983). Scenario planning — what style should you use? Long Range Planning, vol. 20, p. 21-29.

Huss W.R., Honton E.J., (1987). Alternative methods for developing business scenarios, Technological Forecasting and Social Change, vol. 31, 219-238.

InEE - Institut Ecologie et Environnement. (2009). Prospective de l'Institut Ecologie et Environnement du CNRS, Compte rendu des 12 et 13 mai, Rennes, InEE - CNRS.

InSHS - Institut Sciences Humaines et Sociales. (2009). Projet scientifique et organisationnel, Institut Sciences Humaines et Sociales, CNRS.

Joliveau T., Michelin Y., (2001). Modèles d'analyse et de représentation pour la prospective paysagère concertée : deux exemples en zone rurale. Représentations spatiales et développement territorial, Paris, Hermes. p. 239-266. 
Joliveau T., Dupuis B., (2006). Conception et utilisation de visualisations numériques pour la gestion paysagère. Revue Internationale de Géomatique, vol. 16, p. 115-134.

Kolb M., Mas J.F., Galicia L., (2013). Evaluating drivers and transition potential models in a complex landscape in southern Mexico. International Journal of Geographical Information Science, vol. 27, iss. 9, p. 1804-1827.

Kok K, Verburg P., Veldkamp T., (2004). Integrated Assessment of the land system: The future of land use, Land Use Policy, vol. 24, p. 517-520.

Lambin E.-F., Geist H.-J., (2006). Land-Use and Land-Cover Change - Local processes and Global impacts, Global Change - The IGBP Series, Ed. Springer.

Lardon S., Mainguenaud M., Roche S., (2006). Représentations spatiales et participation. Revue Internationale de Géomatique, vol. 16, n², p. 141-145.

Lardon S., Capitaine M., (2008). Chorèmes et graphes : production et transformation de représentations spatiales en agronomie. Revue d'Anthropologie des Connaissances, vol. 2 , p. 195-217.

Lardon S., Roche S., (2008). Représentations spatiales dans les démarches participatives. Production et usages. Revue Internationale de Géomatique, vol. 18, nº 4, p. 423-428.

Leenhardt D., Trouvat J-L., Gonzalès G., Pérarnaud V., Prats V., Bergez J-E., (2004). Estimating irrigation demand for water management on a regional scale. I. ADEAUMIS, a simulation platform based on bio-decisional modelling and spatial information. Agricultural Water Management, vol. 68, iss. 3, p. 207-232.

Marchadier C., Houet T., Bretagne G., (2012). How to define and assess city adaptation strategies? ICUC8 - 8th International Conference on Urban Climate - ICUC 8 and 10th Symposium on the Urban Environment, August 6-10, 2012, Dublin, Ireland.

Mas J.F., Kolb M., Houet T., Paegelow M., Camacho Olmedo M., (2011). Éclairer le choix des outils de simulation des changements des modes d'occupation et d'usages des sols. Une approche comparative. Revue Internationale de Géomatique, vol. 21, n³, p. 405-430

Mas J.F., Kolb M., Paegelow M., Camacho Olmedo MT, Houet T., (2014). Modelling Land use / cover changes: a comparison of conceptual approaches and softwares. Environmental Modelling and Software, vol. 51, p. 94-111.

Meadows D., Meadows D., Randers J. Behrens W., (1972). The Limits to Growth, Universe Books, New York.

Mermet L. (2003). Prospectives pour l'environnement. Quelles recherches? Quelles ressources? Quelles méthodes? Collection Réponses environnement. La Documentation Française.

Michelin Y. (2000). Le bloc-diagramme: une clé de compréhension des représentations du paysage chez les agriculteurs? Mise au point d'une méthode d'enquête préalable à une gestion concertée du paysage en Artense (massif central français), Cybergéo, $\mathrm{n}^{\circ} 118$, http://cybergeo.revues.org/1992

Perret J., Curie F., Gaffuri J., Ruas A., (2010). A Multi-Agent System for the simulation of urban dynamics, Proceeding of the 10th European Conference on Complex Systems, Lisbon, Portugal. http://geopensim.ign.fr/IMG/pdf/ECCS2010.pdf 
Phlipponneau M. (1971). Géographie et perspectives à long terme, Ouvrage de synthèse, IV ${ }^{\text {th }}$ international Symposium of the International Geographical Union, Commission on Applied Geography, Editions Coconnier, Sablé, France.

Piveteau V. (1995). Prospective et territoire : apports d'une réflexion sur le jeu. Cemagref Editions, collection Gestion des Territoires $n^{\circ} 15$

Pontius R.G., Schneider L.C., (2001). Land-cover change model validation by an ROC method for the Ipswich watershed (Massachusetts, USA), Agriculture, Ecosystems \& Environment, vol. 85, p. 239-248.

Pontius R.G. (2002). Statistical methods to partition effects of quantity and location during comparison of categorical maps at multiple resolutions. Photogrammetric Engineering \& Remote Sensing, vol. 68, p. 1041-1049.

Pontius R.G., Huffaker D., Denman K., (2004a). Useful techniques of validation for spatially explicit land-change models. Ecological Modelling, vol. 179, iss. 4, p. 445-461.

Pontius R.G., Shusas E., McEachern M., (2004b). Detecting important categorical land changes while accounting for persistence. Agriculture, Ecosystems \& Environment, vol. 101, p. 251-268.

Pontius R.G., Boersma W., Castella J.C., Clarke K., de Nijs T., Dietzel C., Duan Z., Fotsing E., Goldstein N., Kok K., Koomen E., Lippitt C.D., McConnell W., Sood A.M., Pijankowski B., Pidhadia S., Sweeney S., Trung T.N., Veldkamp A.T., Verburg P., (2008). Comparing the input, output, and validation maps for several models of land change. Annals of Regional Science, vol. 42, iss. 1, p. 11-27.

Poux X. (2003). Les méthodes des scénarios, Prospectives pour l'environnement: Quelles recherches? Quelles ressources? Quelles méthodes?, La documentation française, p. 3350

Schoemaker P.J.H. (1993). Multiple Scenario development: its conceptual and behavioral foundation, Strategic Management Journal, vol. 14, p. 193-213.

Schoemaker P.J.H. (1995). Scenario planning: a tool for strategic thinking, Sloan Management Review, vol. 36, p. 25-40.

Schwartz P. (1996). The art of the long view: planning for the future in an uncertain world, Currency Doubleday, New York.

Turner B., Lambin E., Reenberg A., (2007). The emergence of land change science for global environmental change and sustainability. Proceedings of the National Academy of Science of the USA, vol. 104, n52, p. 20666-20671.

Uuemaa E., Antrop M., Roosaare J., Marja R., Mander Ü., (2009). Landscape metrics and indices: an overview of their use in landscape research, Living Reviews in Landscape Research, $\mathrm{n}^{\circ} 3$, http://www.livingreviews.org/lrlr-2009-1

Van der Leeuw S., Costanza R., Aulenbach S., Brewer S., BurekM., Cornell S., Crumley C., DearingJ.A., Downy C., Graumlich L.J., Heckbert S., Hegmon M., Hibbard K., Jackson S.T., Kubiszewski I., Sinclair P., Sörlin S., Steffen W., 2011, Toward an integrated history to guide the future. Ecology and Society, vol. 16, n 4 , http://eprints.soton.ac.uk/203317/ 
Verburg P., Eickhout B., Van Meijl H., (2008). A multi-scale, multi-model approach for analyzing the future dynamics of European land use. Annals of Regional Science, vol. 42, iss. 1 , p. $57-77$.

Verburg P., van Berkel D., van Doorn A., van Eupen M., van den Heiligenberg H., (2010). Trajectories of land use change in Europe: a model-based exploration of rural futures, Landscape Ecology, vol. 25, p. 217-232.

Voiron C. (2006). L'espace dans la modélisation des interactions nature-société. Actes du colloque International Interactions Nature-Société. La Baule, 3-6 mai 2006.

Voirin C. (2012). L'anticipation du changement en prospective et des changements spatiaux en géoprospective, L'espace Géographique, vol. 12, p. 99-110.

Wack P. (1985a). Scenarios: uncharted waters ahead, Harvard Business Review, vol. 63, p. 73-89.

Wack P. (1985b). Scenarios: shooting the rapids, Harvard Business Review, vol. 63, p. 139150.

Wilson I. (1998). Mental maps of the future: an intuitive logics approach to scenarios. Learning from the Future: Competitive Foresight Scenarios, first ed., John Wiley \& Sons Inc., New York, p. 81-108. 